Article

\title{
Hawking and Unruh Effects of a 5-Dimensional Minimal Gauged Supergravity Black Hole by a Global Embedding Approach
}

\author{
Hui-Hua Zhao ${ }^{1,2, *}$, Li-Chun Zhang ${ }^{2}$ and Guang-Liang $\mathrm{Li}^{1}$ \\ 1 Department of Applied Physics, Xi'an Jiaotong University, Xianning Road, Xi'an 710049, China; \\ E-Mail: Leegl@mail.xjtu.edu.cn \\ 2 Department of Physics and Institute of Theoretical Physics, Shanxi Datong University; \\ Xingyun Street, Datong 037009, China; E-Mail: zhaoren2969@yahoo.com.cn \\ * Author to whom correspondence should be addressed; E-Mail: kietemap@stu.xjtu.edu.cn; \\ Tel.: +86-152-3529-5615; Fax: +86-0352-7158644.
}

Received: 22 January 2013; in revised form: 12 March 2013 / Accepted: 12 March 2013 / Published: 18 March 2013

\begin{abstract}
Using the new global embedding approach we investigate Unruh/Hawking temperature of the 5-dimensional minimal gauged supergravity black hole with double rotating parameters in a general $(1+1)$ space-time. Our results verify that views of Banerjee and Majhi, and extend this approach to a higher dimension situation.
\end{abstract}

Keywords: Unruh temperature; Hawking temperature; global embedding approach; spherically non-symmetric black hole

PACS Codes: 04.70.Dy; 04.62.+v

\section{Introduction}

After the quantum effect of a black hole was interpreted as the event horizons emitting thermal radiation particles[1-5] and the black hole entropy was first studied [6], black hole theory developed from a simple geometric theory into a multidisciplinary theory including quantum theory, relativity, statistics, astrophysical and differential geometry. Because these discoveries closely contact with the mathematic similarities between some laws of black hole physics and general thermodynamic laws, they provide a clear physical explanation for the similarities. Hawking radiation results and other 
deductions can give us the most profound insights about the quantum gravity. Up to now, there are many methods to calculate Hawking radiation and they have been continually discussed and improved [7-14]. In order to better understand the physical mechanism of Hawking radiation, Unruh has proved that in Minkowski space-time when the quantum field is in a general vacuum state, the observer with a straight uniform acceleration will see a thermal state with temperature that is in direct proportion to its internal acceleration $T=a / 2 \pi$, where $a$ is the acceleration of the observer $[4,5,15]$. Then the relation between Hawking and Unruh effect has been extensively studied. A unified picture of the Hawking effect [1,2] and Unruh effect [15] was established by the global embedding of a curved space-time into a higher dimensional flat space [10], and Refs. [10,16,17] have provided internal relations between Hawking effect and Unruh effect. Then studying thermal radiation of a black hole by Unruh effects aroused widespread concern [18-28], but their research are mainly on 4-dimensional space-times. reference [11] has discussed Hawking/Unruh temperature of 4-dimensional spherically non-symmetric Kerr-Newman space-time and they derived a satisfactory conclusion.

Recently reference [12], considering the response function of an Unruh-DeWitt detector is not merely related with space-time but also vacuum state, observed violation of the equivalence principle and found Hawking temperatures are not consistent with Unruh temperatures. However from the paper we can find that Hawking temperature and Unruh temperature have a particular relationship locally, and furthermore the authors studied only the low-dimensional space-time. references. [7,9] show there are some problems with deriving the Unruh temperature via dimensional reduction and the anomaly cancellation. However it was claimed that this inconsistency in the dimensional reduction method to the Unruh effect has been resolved in reference [13], so the Hawking and Unruh effects need further studies.

According to the latest membrane horizon image, the 4-dimensional space-time where we have lived may be a membrane in a high-dimensional space-time. And the discovery of the remarkable AdS/CFT correspondence showed that bulk properties of solutions in five dimensional gauged supergravities are related to properties of strongly coupled conformal field theories on the four-dimensional boundary of five-dimensional anti-de Sitter space-time [29]. As the energy level of quantum gravity can reduce to $\mathrm{TeV}$ order, it is expected that in a Large Hadron Collider (LHC) there are substantial micro black holes [30-32]. This makes it possible to test the Hawking effect and detect extra dimensionality, so researches on high-dimensional black holes, especially high-dimensional rotating black holes, are very meaningful. In this article we mainly study the Unruh and Hawking temperature of the 5-dimensional minimal gauged supergravity black hole by the reduced global embedding approach [11], and our purpose is to generalize and extend this approach to higher dimensional space-time.

The contents of this paper are as follows: in Section 2, we will reduce a 5-dimensional black hole with double rotating parameters to a 2-dimensional spherically symmetric black hole using the dimensional reduction method near the horizon [it is only (t-r)-sector]. In Section 3 we shall find the reduced global embedding model of a general 2-dimensional black hole space-time which is spherically symmetric. The expression of the reduced global embedding model for a Reissner-Nordstrom-Anti de Sitter (RN-AdS) black hole is given in the next section, and this approach will be exploited to find the Unruh/Hawking temperature for the black hole in 5-dimensional minimal gauged supergravity. Section 5 is devoted to presenting our conclusions. 


\section{Dimensional Reduction near the Horizon}

In this section, motivated from the fact that a n-dimensional black hole metric effectively reduces to a 2-dimensioanal metric (only the (t-r)-sector) near the event horizon [7,33-35], we will reduce a 5-dimensional minimal gauged supergravity black hole with double rotating parameters to a 2-dimensional spherically symmetric black hole using the dimensional reduction technique near the horizon.

Chong, Cvetic and Lu provided Kerr-(Anti)-de Sitter metrics of a 5-dimensional black hole [36]. It is a general non-extremal rotating black hole in minimal five-dimensional gauged supergravity, with independent rotation parameters in the two orthogonal 2-planes, whose metrics in general describes a regular rotating black hole, providing the parameters lie in appropriate ranges so that naked singularities and closed timelike curves are avoided. Its line element is:

$$
\begin{gathered}
d s^{2}=-\frac{\Delta_{\theta}}{\Xi_{a} \Xi_{b}}\left(1+g^{2} r^{2}\right) d t^{2}+\frac{2 m}{\rho^{2}}\left(\frac{\Delta_{\theta} d t}{\Xi_{a} \Xi_{b}}-\sigma\right)^{2}+\frac{\rho^{2}}{\Delta_{r}} d r^{2}+\frac{\rho^{2}}{\Delta_{\theta}} d \theta^{2} \\
+\frac{r^{2}+a^{2}}{\Xi_{a}} \sin ^{2} \theta d \phi^{2}+\frac{r^{2}+b^{2}}{\Xi_{b}} \cos ^{2} \theta d \psi^{2}
\end{gathered}
$$

where:

$$
\begin{gathered}
\Delta_{r}=\frac{1}{r^{2}}\left(r^{2}+a^{2}\right)\left(r^{2}+b^{2}\right)\left(1+g^{2} r^{2}\right)-2 m \\
\Delta_{\theta}=1-a^{2} g^{2} \cos ^{2} \theta-b^{2} g^{2} \sin ^{2} \theta \\
\Xi_{a}=1-a^{2} g^{2} \\
\Xi_{b}=1-b^{2} g^{2} \\
\sigma=a \sin ^{2} \theta \frac{d \varphi}{\Xi_{a}}+b \cos ^{2} \theta \frac{d \psi}{\Xi_{b}} \\
\rho^{2}=r^{2}+a^{2} \cos ^{2} \theta+b^{2} \sin ^{2} \theta .
\end{gathered}
$$

The two angular momenta are:

$$
\begin{aligned}
& J_{a}=\frac{2 \pi a m}{4 \Xi_{a}^{2} \Xi_{b}} \\
& J_{b}=\frac{2 \pi b m}{4 \Xi_{b}^{2} \Xi_{a}}
\end{aligned}
$$

in which the rotating parameters $a$ and $b$ are not equal in magnitude to be the non-extremal case. The conserved mass, or energy:

$$
E=\frac{m \pi\left(2 \Xi_{a}+2 \Xi_{b}-\Xi_{a} \Xi_{b}\right)}{4 \Xi_{a}^{2} \Xi_{b}^{2}}
$$

Killing vector is:

$$
\ell=\frac{\partial}{\partial t}+\Omega_{a} \frac{\partial}{\partial \varphi}+\Omega_{b} \frac{\partial}{\partial \psi},
$$

with angular velocities: 


$$
\begin{aligned}
& \Omega_{a}=\frac{a\left(1+g^{2} r_{+}^{2}\right)}{\left(r_{+}^{2}+a^{2}\right)}, \\
& \Omega_{b}=\frac{b\left(1+g^{2} r_{+}^{2}\right)}{\left(r_{+}^{2}+b^{2}\right)} .
\end{aligned}
$$

At the outer Killing horizon of the maximum positive root $r=r_{+}$of equation $\Delta_{r}=0$, Killing vector becomes a zero vector. One can then easily evaluate the surface gravity:

$$
\kappa=\frac{r_{+}^{2} \Delta_{r}^{\prime}\left(r_{+}\right)}{2\left(r_{+}^{2}+a^{2}\right)\left(r_{+}^{2}+b^{2}\right)}=\frac{r_{+}^{4}\left[1+g^{2}\left(2 r_{+}^{2}+a^{2}+b^{2}\right)\right]-a^{2} b^{2}}{r_{+}\left(r_{+}^{2}+a^{2}\right)\left(r_{+}^{2}+b^{2}\right)} .
$$

According to metric (1), we can derive:

$$
\sqrt{-g}=\frac{r \rho^{2} \sin \theta \cos \theta}{\Xi_{a} \Xi_{b}}
$$

Extending the dimensional reduction method proposed by references [33,34] to 5-dimensional space-time, we will consider matter field, a scalar field for simplicity, in the rotating black hole of $D=5$ minimal gauged supergravity background. The action consists of the free part:

$$
S_{\text {free }}=\int d^{5} x \sqrt{-g} g^{\mu v} \partial_{\mu} \phi^{*} \partial_{\nu} \phi .
$$

By substituting both Equations (1), (15) and (16), we obtain:

$$
\begin{gathered}
S_{\text {free }}=\int \frac{r d t d r d \theta d \varphi d \psi \sin \theta \cos \theta}{\Xi_{a} \Xi_{b}} \phi^{*}\left[\rho^{2} g^{t t}\left(\partial_{t}\right)^{2}+2 \rho^{2} g^{t \varphi} \partial_{t} \partial_{\varphi}+2 \rho^{2} g^{t \psi} \partial_{t} \partial_{\psi}+\frac{1}{r} \partial_{r} r \Delta_{r} \partial_{r}\right. \\
\left.+\rho^{2} g^{\varphi \varphi} \partial_{\varphi}^{2}+\rho^{2} g^{\psi \psi} \partial_{\psi}^{2}+\rho^{2} g^{\varphi \psi} \partial_{\varphi} \partial_{\psi}+\frac{1}{\sin \theta \cos \theta} \partial_{\theta} \sin \theta \cos \theta \partial_{\theta}\right] \phi .
\end{gathered}
$$

The transformation of $r$ to tortoise coordinate $r_{*}$ is defined by:

$$
d r_{*}=\frac{\left(r^{2}+a^{2}\right)\left(r^{2}+b^{2}\right)}{r^{2} \Delta_{r}} d r=\frac{1}{f(r)} d r .
$$

After this transformation, the action Equation (17) is written by:

$$
\begin{gathered}
S_{\text {free }}=\int \frac{r d t d r_{*} d \theta d \varphi d \psi \sin \theta \cos \theta}{\Xi_{a} \Xi_{b}} \phi^{*} f(r)\left[\rho^{2} g^{t t}\left(\partial_{t}\right)^{2}+2 \rho^{2} g^{t \varphi} \partial_{t} \partial_{\varphi}+2 \rho^{2} g^{t \psi} \partial_{t} \partial_{\psi}\right. \\
+\frac{1}{r f(r)} \partial_{r_{*}}\left(\frac{\left(r^{2}+a^{2}\right)\left(r^{2}+b^{2}\right)}{r}\right) \partial_{r_{*}}+\rho^{2} g^{\varphi \varphi} \partial_{\varphi}^{2}+\rho^{2} g^{\psi \psi} \partial_{\psi}^{2} \\
\left.+\rho^{2} g^{\varphi \psi} \partial_{\varphi} \partial_{\psi}+\frac{1}{\sin \theta \cos \theta} \partial_{\theta} \sin \theta \cos \theta \partial_{\theta}\right] \phi .
\end{gathered}
$$

Considering this action in the region near horizon, since $f\left(r_{+}\right)=0$ at $r \rightarrow r_{+}$, we only retain dominant term in (19), thus we obtain: 


$$
\begin{gathered}
S_{\text {free }}=\int \frac{r d t d r_{*} d \theta d \varphi d \psi \sin 2 \theta}{2 \Xi_{a} \Xi_{b}} \phi^{*}\left[-\frac{\left(r^{2}+a^{2}\right)\left(r^{2}+b^{2}\right)}{r^{2}}\left(\partial_{t}\right)^{2}\right. \\
-2 a \frac{\left(r^{2}+a^{2}\right)\left(r^{2}+b^{2}\right)\left(1+g^{2} r^{2}\right)}{r^{2}} \partial_{t} \partial_{\varphi}-2 b \frac{\left(r^{2}+a^{2}\right)\left(r^{2}+b^{2}\right)\left(1+g^{2} r^{2}\right)}{r^{2}} \partial_{t} \partial_{\psi} \\
+\frac{1}{r} \partial_{r_{*}}\left(\frac{\left(r^{2}+a^{2}\right)\left(r^{2}+b^{2}\right)}{r}\right) \partial_{r_{*}}-\frac{a^{2}\left(r^{2}+b^{2}\right)\left(r^{2}+g^{2} r^{2}\right)^{2}}{r^{2}\left(r^{2}+a^{2}\right)} \partial_{\varphi}^{2} \\
\left.-\frac{b^{2}\left(r^{2}+a^{2}\right)\left(r^{2}+g^{2} r^{2}\right)^{2}}{r^{2}\left(r^{2}+b^{2}\right)} \partial_{\psi}^{2}-\frac{a b\left(r^{2}+g^{2} r^{2}\right)^{2}}{r^{2}} \partial_{\varphi} \partial_{\psi}\right] \phi .
\end{gathered}
$$

Here $\theta$ runs over the range 0 to $\pi$, and $\varphi, \psi$ take values between 0 and $2 \pi$. Then by expanding the complex field $\phi$ as:

$$
\phi=\sum_{l, m_{a}, m_{b}} \phi_{l, m_{a}, m_{b}}(r, t) P_{l, m_{a}}(\cos \theta) e^{i m_{a} \varphi} e^{i m_{b} \psi}
$$

and substituting Equation (21) into Equation (20), we have:

$$
\begin{gathered}
S_{\text {free }}=\int \frac{d t d r}{4 \Xi_{a} \Xi_{b}} \frac{\left(r^{2}+a^{2}\right)\left(r^{2}+b^{2}\right)}{r} \phi_{l, m_{a}, m_{b}}^{*} \frac{1}{\Delta_{r}}\left[-\frac{\left(r^{2}+a^{2}\right)\left(r^{2}+b^{2}\right)}{r^{2}}\left(\partial_{t}\right)^{2}\right. \\
-2 a \frac{\left(r^{2}+a^{2}\right)\left(r^{2}+b^{2}\right)\left(1+g^{2} r^{2}\right)}{r^{2}} \partial_{t} \partial_{\varphi}-2 b \frac{\left(r^{2}+a^{2}\right)\left(r^{2}+b^{2}\right)\left(1+g^{2} r^{2}\right)}{r^{2}} \partial_{t} \partial_{\psi} \\
+\Delta_{r} \partial_{r}\left(\frac{r^{2} \Delta_{r}}{\left(r^{2}+a^{2}\right)\left(r^{2}+b^{2}\right)}\right) \partial_{r}-\frac{a^{2}\left(r^{2}+b^{2}\right)\left(r^{2}+g^{2} r^{2}\right)^{2}}{r^{2}\left(r^{2}+a^{2}\right)} \partial_{\varphi}^{2}, \\
-\frac{b^{2}\left(r^{2}+a^{2}\right)\left(r^{2}+g^{2} r^{2}\right)^{2}}{r^{2}\left(r^{2}+b^{2}\right)} \partial_{\psi}^{2}-\frac{a b\left(r^{2}+g^{2} r^{2}\right)^{2}}{\left.r^{2} \partial_{\varphi} \partial_{\psi}\right] \phi_{l, m_{a}, m_{b}}} \\
=-\int \frac{d t d r}{4 \Xi_{a} \Xi_{b}} \frac{\left(r^{2}+a^{2}\right)\left(r^{2}+b^{2}\right)}{r} \phi_{l, m_{a}, m_{b}}^{*}\left[\frac{\left(r^{2}+a^{2}\right)\left(r^{2}+b^{2}\right)}{r^{2} \Delta_{r}}\right. \\
\left.\left(\partial_{t}+\frac{i a m_{a}\left(1+g^{2} r^{2}\right)}{\left(r^{2}+a^{2}\right)}+\frac{i b m_{b}\left(1+g^{2} r^{2}\right)}{\left(r^{2}+b^{2}\right)}\right)^{2}-\partial_{r}\left(\frac{r^{2} \Delta_{r}}{\left(r^{2}+a^{2}\right)\left(r^{2}+b^{2}\right)}\right) \partial_{r}\right] \phi_{l, m_{a} m_{b}},
\end{gathered}
$$

where $m_{a}$ and $m_{b}$ are the magnetic quantum numbers.

From this action we find that $\phi_{l, m_{a} m_{b}}$ can be considered as a $(1+1)$-dimensional complex scalar field in the backgrounds of the dilaton $\Phi$, metric $g_{\mu \nu}$ and $U(1)$ gauge field $A_{\mu}$ :

$$
\begin{gathered}
\Phi=-\frac{\left(r^{2}+a^{2}\right)\left(r^{2}+b^{2}\right)}{r 4 \Xi_{a} \Xi_{b}}, \\
g_{t t}=f(r), \\
g_{r r}=-\frac{1}{f(r)},
\end{gathered}
$$




$$
\begin{gathered}
g_{r t}=0 \\
A_{t}=-\frac{a\left(1+g^{2} r^{2}\right)}{\left(r^{2}+a^{2}\right)}-\frac{b\left(1+g^{2} r^{2}\right)}{\left(r^{2}+b^{2}\right)} .
\end{gathered}
$$

From Equations (23)-(27), we find that the 5-dimensional spherically non-symmetric metric Equation (1) behaves as 2-dimensional spherically metric in the region near the horizon.

$$
d s^{2}=f(r) d t^{2}-\frac{1}{f(r)} d r^{2} .
$$

\section{Reduced Global Embedding}

In this section we will find the reduced global embedding model of a general effective 2-dimensional coordinate near horizon and radiation temperature expressions are provided.

It is interesting to see that the $t-r$ coordinate Equation (28) can be globally embedded in a flat space as:

$$
d s^{2}=\left(d z^{0}\right)^{2}-\left(d z^{1}\right)^{2}-d z^{i} d z_{i},
$$

where $i=2,3, \cdots$. Split the region near horizon into external and internal regions with the event horizon [8], the relations among the flat and curved coordinates are:

$$
\begin{gathered}
z_{\text {out }}^{0}=\frac{2}{f^{\prime}\left(r_{+}\right)} f^{1 / 2}(r) \sinh \left(f^{\prime}\left(r_{+}\right) t / 2\right) \\
z_{\text {out }}^{1}=\frac{2}{f^{\prime}\left(r_{+}\right)} f^{1 / 2}(r) \cosh \left(f^{\prime}\left(r_{+}\right) t / 2\right) \\
z_{\text {in }}^{0}=\frac{2}{f^{\prime}\left(r_{+}\right)}[-f(r)]^{1 / 2} \cosh \left(f^{\prime}\left(r_{+}\right) t / 2\right) \\
z_{\text {in }}^{1}=\frac{2}{f^{\prime}\left(r_{+}\right)}[-f(r)]^{1 / 2} \sinh \left(f^{\prime}\left(r_{+}\right) t / 2\right)
\end{gathered}
$$

where $r_{+}$is the horizon location of the black hole and satisfies the equation $f\left(r_{+}\right)=0$. The subscript "in" ("out") stands for the inside (outside) of the event horizon while variables without any suffix imply that they are valid on both sides of the horizon. From Equations (30) and (31), we have:

$$
\begin{aligned}
& d z_{\text {out }}^{0}=\frac{f^{\prime}(r)}{f^{\prime}\left(r_{+}\right)} f^{-1 / 2}(r) \sinh \left(f^{\prime}\left(r_{+}\right) t / 2\right) d r+f^{1 / 2} \cosh \left(f^{\prime}\left(r_{+}\right) t / 2\right) d t, \\
& d z_{\text {out }}^{1}=\frac{f^{\prime}(r)}{f^{\prime}\left(r_{+}\right)} f^{-1 / 2}(r) \cosh \left(f^{\prime}\left(r_{+}\right) t / 2\right) d r+f^{1 / 2} \sinh \left(f^{\prime}\left(r_{+}\right) t / 2\right) d t .
\end{aligned}
$$

According to refence [11], the Hawking temperature detector moving in the curved space outside the event horizon on a constant $r$ surface, maps to the Unruh detector on the constant $\left(z^{2}, z^{3}, z^{i}, \cdots\right)$ surface. The trajectory of the Unruh detector is given by:

$$
\left(z_{\text {out }}^{1}\right)^{2}-\left(z_{\text {out }}^{0}\right)^{2}=\frac{4}{\left[f^{\prime}\left(r_{+}\right)\right]^{2}} f(r)=\frac{1}{\widetilde{a}^{2}},
$$

leading to the local Hawking temperature: 


$$
T=\frac{\hbar \widetilde{a}}{2 \pi}=\frac{\hbar f^{\prime}\left(r_{+}\right)}{4 \pi} f^{-1 / 2}(r)
$$

The observer at point $r=r_{0}$ outside the black hole horizon obtains that Hawking radiation temperature $T_{H}$ satisfies the following equation [37]:

$$
\sqrt{g_{t t}\left(r_{0}\right)} T_{H}=\sqrt{f(r)} T=\frac{\hbar f^{\prime}\left(r_{+}\right)}{4 \pi} .
$$

So:

$$
T_{H}=\frac{\hbar f^{\prime}\left(r_{+}\right)}{4 \pi \sqrt{g_{t t}\left(r_{0}\right)}} .
$$

From Equations (34) and (35), we have:

$$
\left(d z^{0}\right)^{2}-\left(d z^{1}\right)^{2}=f d t^{2}-\left[\frac{f^{\prime}(r)}{f^{\prime}\left(r_{+}\right)}\right]^{2} \frac{d r^{2}}{f(r)}=f d t^{2}-\frac{d r^{2}}{f(r)}+\left[1-\left(\frac{f^{\prime}(r)}{f^{\prime}\left(r_{+}\right)}\right)^{2}\right] \frac{d r^{2}}{f(r)} .
$$

Comparing Equation (40) with Equations (28) and (29), we find:

$$
d z^{i} d z_{i}=\left[1-\left(\frac{f^{\prime}(r)}{f^{\prime}\left(r_{+}\right)}\right)^{2}\right] \frac{d r^{2}}{f(r)} .
$$

\section{Examples}

In the following sub-sections we shall find the global embeddings of effective 2-dimensional coordinates of different metrics near their respective horizons.

\subsection{Schwarzschild Metric}

In the Schwarzschild metric [7,11]:

$$
\begin{gathered}
f(r)=1-\frac{2 m}{r} \\
r_{+}=2 m \\
g_{t t}(r)=f(r)
\end{gathered}
$$

When $r_{0} \rightarrow \infty$, from Equation (39) we can obtain that Hawking radiation temperature by an observer located at infinity is:

$$
T_{H}=\frac{\hbar}{8 \pi m}
$$

Substituting Equations (42) and (43) into Equation (41), we obtain:

$$
z^{2}=\int d r\left(\frac{\left(r^{2}+r_{+}^{2}\right)\left(r+r_{+}\right)}{r^{3}}\right)^{1 / 2}=\int d r\left(1+\frac{r^{2} r_{+}+r r_{+}^{2}+r_{+}^{3}}{r^{3}}\right)^{1 / 2} .
$$

This result is consistent with the result from reference [11]. For the Schwarzschild metric, we only let $i=2$ in Equation (41). 


\section{2. $R N$-AdS Metric}

For the RN-AdS metric:

$$
\begin{gathered}
f(r)=1-\frac{\mu}{r}+\frac{q^{2}}{r^{2}}+\frac{r^{2}}{l^{2}}, \\
g_{t t}=f(r) .
\end{gathered}
$$

Gravity mass $m=\mu / 2$ with:

$$
\mu=r_{+}+r_{-}+\frac{\left(r_{+}^{2}+r_{-}^{2}\right)\left(r_{+}+r_{-}\right)}{l^{2}}
$$

and static charge:

$$
q^{2}=r_{+} r_{-}\left(1+\frac{r_{+}^{3}-r_{-}^{3}}{l^{2}\left(r_{+}-r_{-}\right)}\right)=r_{+} r_{-}\left(1+\frac{r_{+}^{2}+r_{-}^{2}+r_{+} r_{-}}{l^{2}}\right),
$$

where $r_{+}$and $r_{-}$are positive roots of $f(r)=0$, and $r_{+}$is the event horizon radius of the black hole.

Substituting Equations (49) and (50) into Equation (47), we can obtain:

$$
f(r)=\frac{\left(r-r_{+}\right)\left(r-r_{-}\right)}{r^{2}}-\frac{\left(r_{+}^{2}+r_{-}^{2}\right)\left(r_{+}+r_{-}\right) r-r_{+} r_{-}\left(r_{+}^{2}+r_{-}^{2}+r_{+} r_{-}\right)-r^{4}}{l^{2} r^{2}}
$$

From (39), the observer at point $r=r_{0}$ outside the black hole horizon obtains that Hawking radiation temperature is as follows:

$$
T_{H}=\frac{\hbar f^{\prime}\left(r_{+}\right)}{4 \pi \sqrt{1-\mu / r_{0}+q^{2} / r_{0}^{2}+r_{0}^{2} / l^{2}}} .
$$

Substituting Equation (51) into Equation (41), we have:

$$
d s^{2}=\left(d z^{0}\right)^{2}-\left(d z^{1}\right)^{2}-\left(d z^{2}\right)^{2}-\left(d z^{3}\right)^{2}-\left(d z^{4}\right)^{2},
$$

where:

$$
\begin{gathered}
z^{2}=\int d r\left[1+\frac{1}{A l^{6} r^{4} r_{+}^{2}\left[f^{\prime}\left(r_{+}\right)\right]^{2}}\left(r^{2} r_{+}+r_{+}^{3}+r r_{+}^{2}\right)\left(l^{3}+l r_{+}^{2}\right)^{2}\right]^{1 / 2}, \\
z^{3}=\int d r\left[\frac{1}{A l^{6} r r_{+}^{2}\left[f^{\prime}\left(r_{+}\right)\right]^{2}}\left(r^{2}+r_{+}^{2}+r r_{+}^{2}\right)\left(l^{4}+10 r_{+}^{2} l^{2}+9 r_{+}^{4}\right)\right]^{1 / 2}, \\
z^{4}=\int d r\left\{\left[\frac{r_{-}^{2}\left(l^{2}+r_{+}^{2}+r_{-}^{2}+r_{+} r_{-}\right)^{2}}{l^{4} r_{+}^{4}}-\frac{2 r_{-}\left(l^{2}+3 r_{+}^{2}\right)\left(l^{2}+r_{+}^{2}+r_{-}^{2}+r_{+} r_{-}\right)}{l^{4} r_{+}^{3}}-\right] \frac{\left(r^{2}+r_{+}^{2}+r_{+} r\right)}{A l^{2} r\left[f^{\prime}\left(r_{+}\right)\right]^{2}}\right. \\
-\frac{r_{-}\left(l^{2}+r_{+}^{2}+r_{-}^{2}+r_{+} r_{-}\right)}{A l^{2} r^{2}}-\frac{r_{-}^{2}\left(l^{2}+r_{+}^{2}+r_{-}^{2}+r_{+} r_{-}\right)^{2}\left[r^{2}\left(r^{2}+r_{+}^{2}+r r_{+}\right)+4 r_{+}^{4}\right]}{A l^{4} r_{+}^{3} r^{6}\left[f^{\prime}\left(r_{+}\right)\right]^{2}} \\
\left.-\frac{2 r_{-}\left(l^{2}+r_{+}^{2}+r_{-}^{2}+r_{+} r_{-}\right)\left[\left(l^{2} r_{+}^{4}+r_{+}^{6}\right)+\left(l^{2} r_{+}+r^{3}\right)\left(r^{2}+r_{+}^{2}\right)\left(r+r_{+}\right)\right.}{l^{4} r_{+}^{3} r^{5}}\right\}^{1 / 2}
\end{gathered}
$$

with: 


$$
A=\frac{\left(l^{2}+r^{2}+r_{+}^{2}+r r_{+}\right)}{l^{2} r}-\frac{r_{-}\left(l^{2}+r_{+}^{2}+r_{-}^{2}+r_{+} r_{-}\right)}{l^{2} r^{2}} .
$$

When $q=0$, Equation (47) reduces to Schwarzschild-AdS metric. Our results consist with the known results proposed by references. [11,12].

\subsection{5-Dimensional Kerr-(Anti)-de Sitter Metric}

For the 5-dimensional Kerr-(Anti)-de Sitter metric:

$$
f(r)=\left(1+g^{2} r^{2}\right)-\frac{r^{2}\left(r_{+}^{2}+a^{2}\right)\left(r_{+}^{2}+b^{2}\right)}{r_{+}^{2}\left(r^{2}+a^{2}\right)\left(r^{2}+b^{2}\right)}\left(1+g^{2} r_{+}^{2}\right),
$$

where $r_{+}$is the location of the black hole horizon, and is a positive root of equation $f(r)=0$.

From Equation (39), the observer at point $r=r_{0}$ outside the black hole horizon obtains that the Hawking radiation temperature is as follows:

$$
T_{H}=\frac{\hbar f^{\prime}\left(r_{+}\right)}{4 \pi \sqrt{g_{t t}\left(r_{0}\right)}}=\frac{r_{+}^{4}\left[1+g^{2}\left(2 r_{+}^{2}+a^{2}+b^{2}\right)\right]-a^{2} b^{2}}{2 \pi r_{+}\left(r_{+}^{2}+a^{2}\right)\left(r_{+}^{2}+b^{2}\right)} \frac{1}{\sqrt{g_{t t}\left(r_{0}\right)}},
$$

where:

$$
\begin{gathered}
g_{t t}\left(r_{0}\right)=\Delta_{\theta}\left(\frac{\rho_{0}^{2} \Xi_{a} \Xi_{b}\left(1+g^{2} r_{0}^{2}\right)-2 m \Delta_{\theta}}{\rho_{0}^{2} \Xi_{a}^{2} \Xi_{b}^{2}}\right), \\
\rho_{0}^{2}=r_{0}^{2}+a^{2} \cos ^{2} \theta+b^{2} \sin ^{2} \theta
\end{gathered}
$$

When $r_{0} \rightarrow \infty$, we can obtain Hawking radiation temperature got by an observer located at infinite:

$$
T_{0}=\frac{r_{+}^{4}\left[1+g^{2}\left(2 r_{+}^{2}+a^{2}+b^{2}\right)\right]-a^{2} b^{2}}{2 \pi r_{+}\left(r_{+}^{2}+a^{2}\right)\left(r_{+}^{2}+b^{2}\right)} .
$$

Substituting Equation (58) into Equation (41), we obtain:

$$
d s^{2}=\left(d z^{0}\right)^{2}-\left(d z^{1}\right)^{2}-\left(d z^{2}\right)^{2}-\left(d z^{3}\right)^{2},
$$

where:

$$
\begin{gathered}
z^{2}=\int d r\left(1+\frac{4 r^{2}\left(1+g^{2} r_{+}^{2}\right)^{2}}{\left[f^{\prime}\left(r_{+}\right)\right]^{2} f(r)} \frac{\left(r_{+}^{4}-a^{2} b^{2}\right)^{2}}{r_{+}^{4}\left(r^{2}+a^{2}\right)\left(r^{2}+b^{2}\right)}\left[\frac{1}{\left(r_{+}^{2}+a^{2}\right)\left(r_{+}^{2}+b^{2}\right)}\left(1+g^{2} r_{+}^{2}\right)\right.\right. \\
\left.\left.-\frac{\left(r_{+}^{2}+a^{2}\right)^{2}\left(r_{+}^{2}+b^{2}\right)^{2}}{\left(r^{2}+a^{2}\right)^{3}\left(r^{2}+b^{2}\right)^{3}}\right]\right)^{1 / 2}, \\
z^{3}=\int d r\left(\frac { 4 g ^ { 2 } r ^ { 2 } ( 1 + g ^ { 2 } r _ { + } ^ { 2 } ) } { [ f ^ { \prime } ( r _ { + } ) ] ^ { 2 } f ( r ) } \left[\frac{g^{2}\left(r_{+}^{2}+a^{2}\right)\left(r_{+}^{2}+b^{2}\right)}{\left(r^{2}+a^{2}\right)\left(r^{2}+b^{2}\right)}-\frac{2 g^{4} r^{2}\left(r_{+}^{4}-a^{2} b^{2}\right)}{\left(r_{+}^{2}+a^{2}\right)\left(r_{+}^{2}+b^{2}\right)}\right.\right. \\
-\frac{2\left(r_{+}^{2}+a^{2}\right)\left(r_{+}^{2}+b^{2}\right)\left(r^{4}-a^{2} b^{2}\right)}{r_{+}^{2}\left(r^{2}+a^{2}\right)^{2}\left(r^{2}+b^{2}\right)^{2}}-\frac{\left(r_{+}^{4}-a^{2} b^{2}\right)^{2}}{r_{+}^{2}\left(r_{+}^{2}+a^{2}\right)^{2}\left(r_{+}^{2}+b^{2}\right)^{2}}
\end{gathered}
$$




\section{Conclusions}

In this paper, using the reduced global embedding approach we obtain the Unruh/Hawking temperature of the 5-dimensional black hole with double rotating parameters in a general $(1+1)$ space-time. Comparing with other methods, we only need investigate the properties of $(1+1)$-dimensional space-time near the horizon, so the calculation is relatively simple, and the application of global embedding approach will promote comprehensive understanding of black holes. It provides a theoretical basis for research on Unruh/Hawking temperatures of complex and dynamic space-time.

\section{Acknowledgments}

We are grateful to Ren Zhao for his various discussions. This work is supported by NSFC under Grant Nos. (11175109; 11075098; 11205097; 11247261) and the Doctoral Sustentation Foundation of Shanxi Datong University (2011-B-03).

\section{References}

1. Hawking, S.W. Black hole explosions? Nature 1974, 248, 30-31.

2. Hawking, S.W. Particle creation by black holes. Commun. Math. Phys. 1975, 43, 199-220.

3. Wald, R.M. On particle creation by black holes. Commun. Math. Phys. 1975, 45, 9-34.

4. Fulling, S.A. Nonuniqueness of canonical field quantization in qiemannian space-time. Phys. Rev. D 1973, 7, 2850-2862.

5. Davies, P.C.W. Scalar production in Schwarzschild and Rindler metrics. J. Phys. A Math. Gen. 1975, 8, 609-616.

6. Bekenstein, J.D. Black Holes and Entropy. Phys. Rev. D 1973, 7, 2333-2346.

7. Robinson, S.P.; Wilczek, F. Relationship between Hawking radiation and gravitational anomalies. Phys. Rev. Lett. 2005, 95, 011303-01136.

8. Akhmedov, E.T.; Piling T.; Singleton, D. Subtleties in the quasi-classical calculation of Hawking radiation. Int. J. Mod. Phys. D 2008, 17, 2453-2458.

9. Akhmedova, V.; Piling, T.; Gill, A.D.; Singleton, D. Comments on an anomaly versus WKB/tunneling methods for calculating Unruh radiation. Phys. Lett. B. 2009, 673, 227-231.

10. Deser, S, Levin, O. Equivalence of Hawking and Unruh temperatures and entropies through flat space embeddings. Class. Quant. Grav. 1998, 15, L85-L88.

11. Banerjee, R.; Majhi, B.R. A new global embedding approach to study Hawking and Unruh effects. Phys. Lett. B 2010, 690, 83-86.

12. Singleton, D.; Wilburn, S. Hawking radiation, Unruh radiation and the equivalence principle. Phys. Rev. Lett. 2011, 107, 081102-081106.

13. Zampeli, A.; Singleton, D.; Vagenas, E.C. Hawking radiation, chirality, and the principle of effective theory of gravity. J. High Energy Phys. 2012, 06, 097-104.

14. Raffaelli, B. A scattering approach to some aspects of the Schwarzschild Black Hole. J. High Energy Phys. 2013, 1301, 188-202.

15. Unruh, W.G. Notes on black-hole evaporation. Phys. Rev. D 1976, 14, 870-992. 
16. Deser, S.; Levin, O. Accelerated detectors and temperature in (anti-) de Sitter spaces. Class. Quant. Grav. 1997, 14, L163-L168.

17. Deser, S, Levin, O. Mapping hawking into unruh thermal properties. Phys. Rev. D 1999, 59, 064004-064013.

18. Hong, S.T.; Kim, W.T.; Oh, J.J.; Park, Y.J. Higher dimensional flat embeddings of black strings in $2+1$ dimensions. Phys. Rev. D 2001, 63, 127502-127505.

19. Santos, N.L.; Dias, O.J.C.; Lemos, J.P.S. Global embedding of $D$-dimensional black holes with a cosmological constant in Minkowskian spacetimes: matching between hawking temperature and Unruh temperature. Phys. Rev. D. 2004, 70, 124033-124039.

20. Chen, H.Z.; Tian, Y. Note on the generalization of the global embedding Minkowski spacetime approach. Phys. Rev. D 2005, 71, 104008-104011.

21. Chen, H.Z; Song, X.C; Tian, Y.; Gao, Y.H. The GEMS approach to stationary motions in the spherically symmetric spacetimes. J. High Energy Phys. 2004, 10, 011.

22. Tian, Y. De Sitter thermodynamics from diamonds's temperature. J. High Energy Phys. 2005, 6, 045.

23. Brynjolfsson, E.J.; Thorlacius, L. Taking the temperature of a black hole. J. High Energy Phys. 2008, 9, 066.

24. Hirayama, T.; Kao, P.W.; Kawamoto, S.; Lin, F.L. Unruh effect and holography. Nucl. Phys. B 2011, 844, 1-25.

25. Caceres, E.; Chernico, M.; Guijosa, A.; Pedraza, J.F. Quantum fluctuations and the Unruh effect in strongly-coupled conformal field theories. J. High Energy Phys. 2010, 6, 78.

26. Crispino, L.C.B.; Higuchi, A.; Matsas, G.E.A. The Unruh effect and its applications. Rev.Mod. Phys. 2008, 80, 787-838.

27. Zhang, L.C.; Li, H.F.; Zhao, R. Hawking and Unruh effects of the cosmological horizon in a higher-dimensional Kerr-de Sitter spacetime by the global embedding approach. Europhys. Lett. 2011, 94, 40003.

28. Zhang, L.C.; Li, H.F.; Zhao, R. Tunneling mechanism in higher-dimensional rotating black hole with a cosmological constant in the approach of dimensional reduction. Astophys. Space Sci. 2011, 333, 457-462.

29. Witten, E. Anti-de Sitters space and holography. Adv. Theor. Math. 1998, 2, 253.

30. Giddings, S.; Thomas, S. High energy colliders as black hole factories: The end of short distance physics. Phys. Rev. D 2002, 65, 056010-056021.

31. Mureika, J.; Nicolini, P.; Spallucci, E. Could any black holes be produced at the LHC? Phys. Rev. D 2012, 85, 106007-106014.

32. Dimopoulos, S.; Landsberg, G. Black holes at the large Hadron collider. Phys. Rev. Lett. 2001, 87, 161602-161605.

33. Iso, S.; Umetsu, H.; Wilczek, F. Anomalies, Hawking radiations, and regularity in rotating black holes. Phys. Rev. D 2006, 74, 044017-044016.

34. Umetsu, K. Hawking radiation from Kerr-Newman black hole and tunneling mechanism. Int. J. Mod. Phy. A. 2010, 25, 4123-4140.

35. Porfyriadis, A.P. Hawking radiation via anomaly cancellation for the black holes of five-dimensional minimal gauged supergravity. Phys. Rev. D 2009, 79, 084039-089045. 
36. Chong Z.W.; Cvetic, M.; Lu, H.; Pope, C.N. General nonextremal rotating black holes in minimal five-dimensional gauged supergravity. Phys. Rev. Lett. 2005, 95, 161301-161304.

37. Tolman, R.C. Relativity, Thermodynamics and Cosmology; Dover Pulication: New York, NY, USA, 1987; p. 318.

(C) 2013 by the authors; licensee MDPI, Basel, Switzerland. This article is an open access article distributed under the terms and conditions of the Creative Commons Attribution license (http://creativecommons.org/licenses/by/3.0/). 